\title{
HEAT CONDUCTION IN A THIN CIRCULAR PIPE DUE TO A CIRCUMFERENTIALLY MOVING HEAT SOURCE*
}

\author{
BY \\ S. NAIR \\ Illinois Institute of Technologv
}

\begin{abstract}
The solution of the title problem is often approximated by the temperature distribution in a flat plate due to an array of periodically placed heat sources, where the curvature of the pipe is neglected. Here, using an asymptotic expansion in terms of the thickness to radius ratio, linear corrections to the flat plate solution are obtained explicitly. Results indicate that these corrections may be significant depending, of course, on the above small parameter and the speed of the moving source.
\end{abstract}

Introduction. There are a number of important engineering problems in which the temperature distributions due to heat sources moving with respect to the medium play a significant role. In calculating the residual stresses in circumferential welds the effect of the weld torch is often modeled as a moving heat source. Wells [1,2] used the solution given in Carslaw and Jaegar [3] for a point source moving on top of a flat plate to study residual stresses in butt-welded plates. This approach was extended to cylindrical pipes by Vaidyanathan et al. $[4,5]$ by conceptually flattening the cylinder into a plate and using an array of sources marching on the plate. This way the periodic appearance of the source at the starting point is taken into account. However, two other features of the original problem are neglected. One of these is the effect of the curvature of the pipe and the other is the fact that a circular pipe, unlike a plate, does not reach a steady state if there is no heat loss from its surface. Our objective here is to obtain the effect of curvature of the pipe and as in the previous studies [4,5], we neglect the transient effects. For this purpose, the solution of the steady state heat conduction equation in moving coordinates applicable for a circular, hollow cylinder is sought in the form of an asymptotic series with the cylinder thickness to the outer radius ratio as a small parameter, $\varepsilon$. Only linear terms in this expansion are presented here. Although higher-order terms may be obtained using the formulation given below, the complexity of the algebra makes it less rewarding as an approach for the solution for thick cylinders.

* Received January 4, 1984. 
Numerical values of the flat plate solution as well as the linear curvature corrections are computed. These results indicate that the corrected solution may be as large as $(1+0.5 \varepsilon)$ times the flat plate solution.

Problem formulation. In cylindrical coordinates $(r, \theta, z)$ the transient heat conduction equation is given by

$$
\frac{\partial^{2} T}{\partial r^{2}}+\frac{1}{r} \frac{\partial T}{\partial r}+\frac{1}{r^{2}} \frac{\partial^{2} T}{\partial \theta^{2}}+\frac{\partial^{2} T}{\partial z^{2}}-\frac{1}{\kappa} \frac{\partial T}{\partial t}+\frac{q}{k}=0,
$$

where $T, q, \kappa$, and $k$ represent the temperature, heat flux into the body, the diffusivity of the body, and the conductivity, respectively. For the present problem, the domain is given by $a<r<b, 0<\theta<2 \pi$ and $-\infty<z<\infty$. In order to render stationary a heat source moving with speed $V$ on the outer surface $r=b$ in the plane $z=0$, we set

$$
\theta=\phi+V t / b .
$$

The heat conduction equation in the moving coordinate system is given by

$$
\frac{\partial^{2} T}{\partial r^{2}}+\frac{1}{r} \frac{\partial T}{\partial r}+\frac{1}{r^{2}} \frac{\partial^{2} T}{\partial \phi^{2}}+\frac{\partial^{2} T}{\partial z^{2}}+\frac{V}{b \kappa} \frac{\partial T}{\partial \phi}+\frac{q}{k}=0,
$$

where we have neglected the term $\partial T / \partial t$ under the assumption of a steady state temperature distribution in the moving frame of reference.

A given heat flux of strength $q_{0}$ at $r=b, \phi=0$ and $z=0$ can be conveniently represented by a source of strength $2 q_{0}$ using

$$
q=2 q_{0} \delta_{3}(r-b, \phi, z),
$$

where the three-dimensional delta function at the boundary, $r=b$, satisfies the relation

$$
\int_{a}^{b} \int_{0}^{2 \pi} \int_{-\infty}^{\infty} \delta_{3} r d r d \phi d x=\frac{1}{2} .
$$

With the assumption that the thickness, $h \equiv b-a$ is small compared to the radius $b$, we introduce the parameter,

$$
\varepsilon=h / b
$$

and the nondimensional quantities

$$
\begin{aligned}
\xi_{1} & =(r-b) / h, \quad \xi_{2}=b \phi / h, \quad \xi_{3}=z / h, \\
\psi & =e^{\alpha \xi_{2}} \tau, \quad \tau=T k h / q_{0}, \quad \delta_{* 3}=\delta_{3} / h^{3}, \quad \alpha=V h / 2 \kappa .
\end{aligned}
$$

The differential equation (3) and the associated boundary conditions can be written as

$$
\begin{aligned}
\psi_{, 11}+\left(1+\varepsilon \xi_{1}\right)^{-2} \psi,_{22}+\psi,,_{33}+\varepsilon\left(1+\varepsilon \xi_{1}\right)^{-1} \psi,_{1}-\alpha^{2} \psi=-2 \delta_{*_{3}} e^{\alpha \xi_{2}}, \\
\psi_{, 1}=0 ; \quad \xi_{1}=0 ; \quad \xi_{2}^{2}+\xi_{3}^{2} \neq 0 \\
\psi_{, 1}=0 ; \quad \xi_{1}=-1 \\
|\psi| \rightarrow 0 ; \quad\left|\xi_{3}\right| \rightarrow \infty .
\end{aligned}
$$

With $\varepsilon$ being a small parameter, we introduce the expansion

$$
\psi=\psi_{0}+\varepsilon \psi_{1}+\cdots
$$


into equation (8) to obtain

$$
\begin{aligned}
& \left(\nabla^{2}-\alpha^{2}\right) \psi_{0}=-2 \delta_{* 3} 2 e^{\alpha \xi_{2} / 2} \\
& \left(\nabla^{2}-\alpha^{2}\right) \psi_{1}=2 \xi_{1} \psi_{0,22}-\psi_{0,1} .
\end{aligned}
$$

The function $\psi_{0}$ represents the temperature distribution due to a moving source on a flat plate of thickness $h$ and $\psi_{1}$ represents a correction to $\psi_{0}$ due to the curvature. Solution of equation (10) is given in [3] using the transient solution for an infinite medium. We may obtain the steady state solutions of equations (10) and (11) more directly using the two-dimensional Fourier transformation with respect to $\xi_{2}$ and $\xi_{3}$. Defining the transformation

$$
\Psi \equiv \mathscr{F}[\psi]=\frac{1}{2 \pi} \int_{-\infty}^{\infty} \int_{-\infty}^{\infty} \psi\left(\xi_{1}, \xi_{2}, \xi_{3}\right) e^{-i\left(\eta_{2} \xi_{2}+\eta_{3} \xi_{3}\right)} d \xi_{2} d \xi_{3},
$$

equations (10) and (11) are written as

$$
\begin{aligned}
& \Psi_{0}^{\prime \prime}-\lambda^{2} \Psi_{0}=-\frac{1}{\pi} \delta_{*_{1}}\left(\xi_{1}\right) ; \quad-1<\xi_{1}<0, \\
& \Psi_{1}^{\prime \prime}-\lambda^{2} \Psi_{1}=-2 \xi_{1} \eta_{2}^{2} \Psi_{0}-\Psi_{0}^{\prime},
\end{aligned}
$$

where

$$
\lambda^{2}=\alpha^{2}+\eta_{2}^{2}+\eta_{3}^{2}, \quad()^{\prime} \equiv()_{1} .
$$

Solutions of equations (13) and (14) are given by

$$
\begin{aligned}
\Psi_{0}\left(\xi_{1} ; \lambda\right)= & \frac{1}{2 \pi} \frac{\operatorname{Cosh} \lambda\left(1+\xi_{1}\right)}{\lambda \operatorname{Sinh} \lambda} ; \\
\Psi_{1}\left(\xi_{1} ; \lambda\right)= & \left\{-2 \pi \eta_{2}^{2} \Psi_{0}(0 ; \lambda)+\xi_{1}\left(\frac{\eta_{2}^{2}}{\lambda^{2}}-\frac{1}{2}\right)\right\} \Psi_{0}\left(\xi_{1} ; \lambda\right) \\
& +\left\{\frac{\eta_{2}^{2}}{\lambda^{2}}\left(1-\xi_{1}^{2}\right)-\frac{\eta_{2}^{2}}{\lambda^{4}}+\frac{1}{2 \lambda^{2}}\right\} \Psi_{0}^{\prime}\left(\xi_{1} ; \lambda\right) .
\end{aligned}
$$

Inversion of Fourier transforms. The functions $\Psi$ are obtained from the integral

$$
\psi=\mathscr{F}^{-1}[\Psi]=\frac{1}{2 \pi} \int_{-\infty}^{\infty} \int_{-\infty}^{\infty} \Psi\left(\xi_{1} ; \lambda\right) e^{i\left(\eta_{2} \xi_{2}+\eta_{3} \xi_{3}\right)} d \eta_{1} d \eta_{2} .
$$

Introducing the variables $\xi, \eta, \phi$, and $\Phi_{1}$ through

$$
\begin{aligned}
& \xi_{2}=\xi \operatorname{Cos} \phi, \quad \eta_{2}=\eta \operatorname{Cos} \phi_{1}, \\
& \xi_{3}=\xi \operatorname{Sin} \phi, \quad \eta_{3}=\eta \operatorname{Sin} \phi_{1},
\end{aligned}
$$

the integral (18) can be rewritten as

$$
\psi=\int_{0}^{\infty} J_{0}(\xi \eta) \Psi\left(\xi_{1} ; \lambda\right) \eta d \eta,
$$

where $J_{0}$ is the Bessel function of order zero. The parameter $\lambda$ in equation (16) is now given by

$$
\lambda=\sqrt{\alpha^{2}+\eta^{2}} .
$$


The integral (20) is evaluated using contour integration. Introducing the relations

$$
\begin{aligned}
& \eta= \pm i \zeta, \quad \mu=\left(\zeta^{2}-\alpha^{2}\right)^{1 / 2} \\
& J_{0}( \pm i \xi \zeta)= \pm \frac{2}{\pi i} K_{0}(\xi \zeta) \mp Y_{0}( \pm i \xi \zeta)
\end{aligned}
$$

where $Y_{0}$ and $K_{0}$ are the Bessel function and modified Bessel function of the second kind, we obtain

$$
\psi=\frac{1}{\pi i} \int_{-i \infty}^{i \infty} \zeta K_{0}(\xi \zeta) \Psi\left(\xi_{1} ; i \mu\right) d \zeta .
$$

By closing the contour using a semicircle on the right side of the imaginary axis, $\psi$ can be written in terms of the residues $R$ of the integrand, in the form

$$
\psi=-2 \sum R\left(\zeta_{n}\right),
$$

where $\zeta_{n}$ are the poles of the function inside the integral sign.

The inversion procedure becomes more tractable if we introduce the relations

$$
\begin{aligned}
& f_{1}=\Psi_{0}(\xi ; i \mu), \quad f_{2}=-2 \pi \Psi_{0}(0 ; i \mu) \Psi_{0}\left(\xi_{1} ; i \mu\right), \\
& f_{3}=-\Psi_{0}\left(\xi_{1} ; i \mu\right) / \mu^{2}, \quad f_{4}=\Psi_{0}\left(\xi_{1} ; i \mu\right) / \mu^{4}, \\
& F_{i}=\mathscr{F}^{-1}\left[f_{i}\right] ; \quad i=1, \ldots, 4 .
\end{aligned}
$$

We write equations (16) and (17) in the form

$$
\begin{aligned}
& \Psi_{0}\left(\xi_{1} ; i \mu\right)=f_{1}, \\
& \Psi_{1}\left(\xi_{1} ; i \mu\right)=\eta_{2}^{2}\left[f_{2}+\xi_{1} f_{3}+\left(1-\xi_{1}^{2}\right) f_{3}^{\prime}-f_{4}^{\prime}\right]+\frac{1}{2} f_{3}^{\prime}-\frac{\xi_{1}}{2} f_{1},
\end{aligned}
$$

and their inverse Fourier transforms

$$
\begin{aligned}
& \Psi_{0}\left(\xi_{1}\right)=F_{1}, \\
& \Psi_{1}\left(\xi_{1}\right)=-\frac{\partial^{2}}{\partial \xi_{2}^{2}}\left[F_{2}+\xi_{1} F_{3}+\left(1-\xi_{1}^{2}\right) F_{3}^{\prime}-F_{4}^{\prime}\right]+\frac{1}{2} F_{3}^{\prime}-\frac{\xi_{1}}{2} F_{1} .
\end{aligned}
$$

From equation (23) we note that in order to find $F_{i}$, we need the residues of the functions,

$$
\begin{aligned}
& \zeta K_{0}(\zeta \xi) f_{1}=\frac{-1}{2 \pi} \zeta K_{0}(\zeta \xi) \frac{\operatorname{Cos} \mu\left(1+\xi_{1}\right)}{\mu \operatorname{Sin} \mu} \\
& \zeta K_{0}(\zeta \xi) f_{2}=\frac{-1}{2 \pi} \zeta K_{0}(\zeta \xi) \frac{\operatorname{Cos} \mu \operatorname{Cos} \mu\left(1+\xi_{1}\right)}{\mu^{2} \operatorname{Sin}^{2} \mu} \\
& \zeta K_{0}(\zeta \xi) f_{3}=\frac{1}{2 \pi} \zeta K_{0}(\zeta \xi) \frac{\operatorname{Cos} \mu\left(1+\xi_{1}\right)}{\mu^{3} \operatorname{Sin} \mu} \\
& \zeta K_{0}(\zeta \xi) f_{4}=-\frac{1}{2 \pi} \zeta K_{0}(\zeta \xi) \frac{\operatorname{Cos} \mu\left(1+\xi_{1}\right)}{\mu^{5} \operatorname{Sin} \mu}
\end{aligned}
$$

These functions have poles at

$$
\zeta_{n}=\sqrt{\alpha^{2}+n^{2} \pi^{2}}, \quad n=0,1, \ldots
$$


Denoting the residues by $R_{i}\left(\zeta_{n}\right)$ we calculate

$$
\begin{gathered}
R_{1}\left(\zeta_{0}\right)=-\frac{1}{4 \pi} K_{0}\left(\xi \zeta_{0}\right), \quad R_{1}\left(\zeta_{n}\right)=-\frac{1}{2 \pi} K_{0}\left(\xi \zeta_{0}\right) \operatorname{Cos} n \pi \xi_{1} \\
R_{2}\left(\zeta_{0}\right)=-\frac{1}{8 \pi \zeta_{0}}\left\{\xi K_{0}^{\prime}\left(\zeta \zeta_{n}\right)-\left[\frac{1}{3}+\left(1+\xi_{1}\right)^{2}\right] \alpha K_{0}\left(\xi \zeta_{n}\right)\right\} \\
R_{2}\left(\zeta_{n}\right)=-\frac{1}{2 \pi \zeta_{n}}\left\{K_{0}^{\prime}\left(\xi \zeta_{n}\right)+\left[\frac{1}{\zeta_{n}}-\frac{2 \zeta_{n}}{n^{2} \pi^{2}}\right] K_{0}\left(\xi \zeta_{n}\right)\right\} \operatorname{Cos} n \pi \xi_{1}, \\
R_{3}\left(\zeta_{0}\right)=\frac{1}{8 \pi \zeta_{0} d}\left\{\xi K_{0}^{\prime}\left(\xi \zeta_{0}\right)+\left[\frac{1}{3}-\left(1+\xi_{1}\right)^{2}\right] \alpha K_{0}\left(\xi \zeta_{0}\right)\right\} \\
R_{3}\left(\zeta_{n}\right)=\frac{1}{2 \pi(n \pi)^{2}} K_{0}\left(\xi \zeta_{n}\right) \operatorname{Cos} n \pi \xi_{1}, \\
R_{4}\left(\zeta_{0}\right)=-\frac{1}{16 \pi \zeta_{0}^{2}}\left\{K_{0}\left(\xi \zeta_{0}\right)\left[\frac{\xi^{2}}{2}+\frac{7 \alpha^{2}}{90}+\frac{\alpha^{2}}{6}\left(1+\xi_{1}\right)^{4}\right]^{7}\right. \\
R_{4}\left(\zeta_{n}\right)=-\frac{1}{2 \pi(n \pi)^{4}} K_{0}\left(\xi \zeta_{n}\right) \operatorname{Cos} n \pi \xi_{1} .
\end{gathered}
$$

Using equations (24) and (28), first we obtain the well-known solution for a flat plate,

$$
\psi_{0}=\frac{1}{2 \pi} \sum_{n=0}^{\infty} a_{n} \operatorname{Cos} n \pi \xi_{1},
$$

where

$$
a_{0}=K_{0}\left(\xi \zeta_{0}\right), \quad a_{n}=2 K_{0}\left(\xi \zeta_{n}\right) .
$$

The function $\psi_{1}$ is obtained using equation (29) in the form

$$
\psi_{1}=\frac{1}{2 \pi} \sum_{n=0}^{\infty} b_{n} \operatorname{Cos} n \pi \xi_{1}+\frac{1}{2 \pi} \sum_{n=1}^{\infty} c_{n} \operatorname{Sin} n \pi \xi_{1},
$$

where

$$
\begin{aligned}
& b_{0}=\frac{1}{2} K_{0}-\frac{1}{\alpha}\left(\xi K_{0}^{\prime}\right), 22+\frac{1+\xi_{1}}{2} K_{0,22}\left[\frac{1}{3}-\left(1-\xi_{1}^{2}\right)+\frac{1}{3}\left(1+\xi_{1}\right)^{2}\right], \\
& b_{n}=-\xi_{1} K_{0}-\frac{2}{\zeta_{n}}\left(\xi K_{0}^{\prime}\right), 22-2 K_{0,22}\left(\frac{1}{\zeta_{n}^{2}}-\frac{2+\xi_{1}}{n^{2} \pi^{2}}\right), \\
& c_{n}=\frac{K_{0}}{n \pi}-2 K_{0,22}\left[\frac{1-\xi_{1}^{2}}{n \pi}+\frac{1}{n^{3} \pi^{3}}\right],
\end{aligned}
$$

with

$$
\begin{aligned}
\left(\xi K_{0}^{\prime}\right)_{, 22} & =\xi_{n} K_{0}-\frac{\xi_{n}^{2} \xi_{2}^{2}}{\xi} K_{1}, \\
K_{0,22} & =\frac{\zeta_{n}^{2} \xi_{2}^{2}}{\xi^{2}} K_{0}-\frac{\xi_{3}^{2}-\xi_{2}^{2}}{\xi^{3}} \zeta_{n} K_{1} .
\end{aligned}
$$

Here, $K_{1}$ represents the modified Bessel function of the second kind of order one. 
Results and discussion. In Fig. 1, a plot of $\psi_{0}$ and $\psi_{1}$ are shown when $\alpha=0.25$ on the outer surface of the cylinder in the plane $\xi_{3}=0$. As discussed in [4], $\alpha=0.25$ is a typical value for welding operations. Since the solution $\psi_{0}$ for a flat plate has a logarithmic singularity as $\xi \rightarrow 0, \psi_{1}$ exhibits $\xi^{-2}$ type singularity close to the source. From Fig. 1 it is clear that the asymptotic solution presented here is not valid when $\xi<0.3$. Beyond this range, depending on the value of $\varepsilon=h / b, \psi_{1}$ may contribute significant corrections to the value of $\psi_{0}$. This figure also shows when $\alpha$ is small, that is, when the speed of the source is low, the effect of curvature is also low on the circumferential temperature distribution. However, as shown in Fig. 2, the contribution of $\psi_{1}$ in the axial direction remains proportional to $\psi_{0}$, independent of $\alpha$. The variations of $\psi_{0} e^{-\alpha \xi_{2}}$ and $\psi_{1} e^{-\alpha \xi_{2}}$ across the thickness of the cylinder are shown in Fig. 3 for $\alpha=0.25, \xi_{2}=0$ and $\xi_{2}=0.3$ when $\xi_{3}=0.3$.

In computing the temperatures, it was found that over 15 terms are required in the series representations (33) and (35) for adequate convergence. As noted earlier, to obtain the temperature distribution due to a source moving around the cylinder, we have to

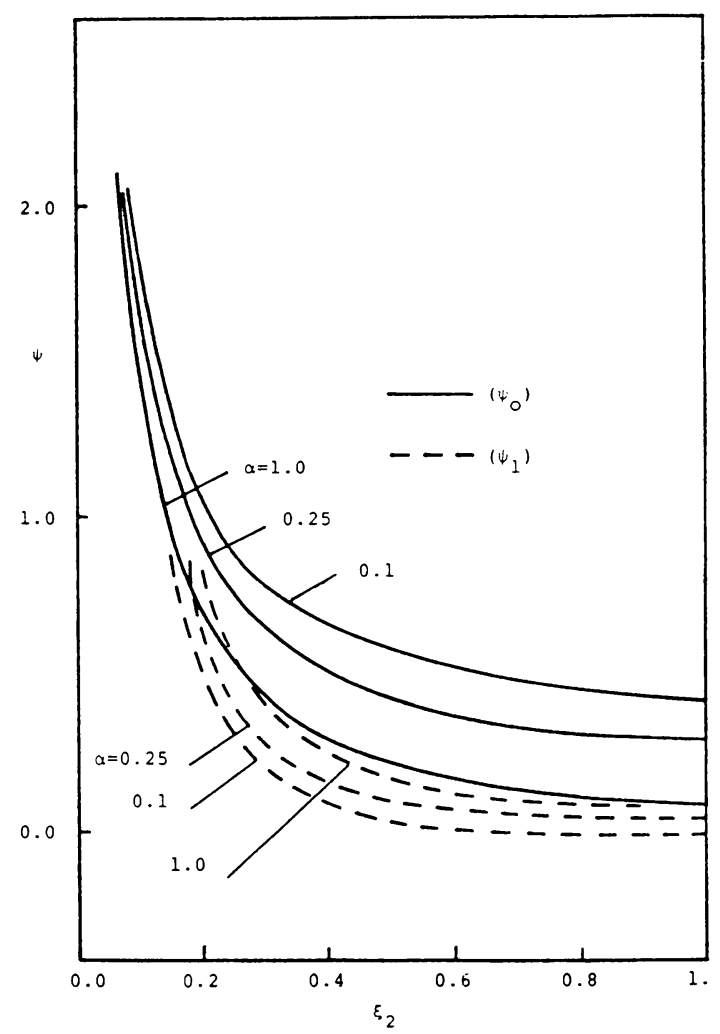

FIG. 1. Circumferential distribution of temperature for different values of $\alpha$. 


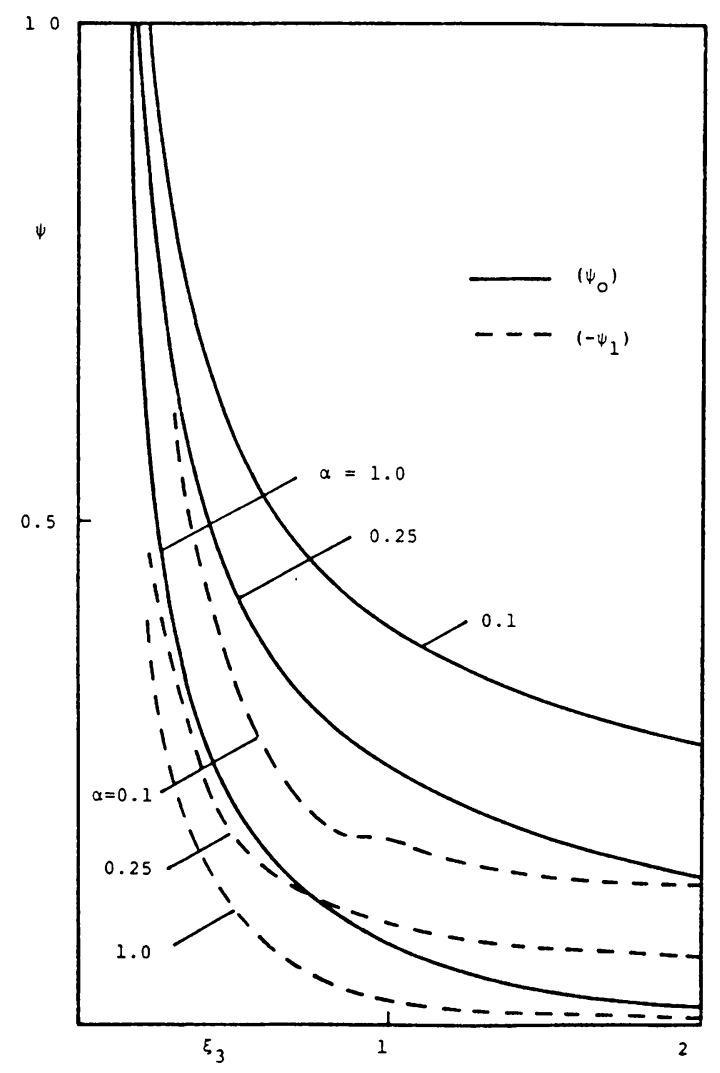

FIG. 2. Axial distribution of temperature for different values of $\alpha$.

consider a series of sources placed on the line $-\infty<\xi_{2}<\infty$ at intervals of $2 \pi b / h$. Fig. 4 shows the nondimensional temperature distribution, $\tau$ at the inner and outer surfaces of the cylinder at $\xi_{3}=0$ when $\alpha=0.25$ and $\varepsilon=0.1$. In this particular case, the temperature rise is due to the source at $\xi_{2}=0$. The contributions due to the sources at $\xi_{2}=2 \pi n / \varepsilon$, $n= \pm 1, \pm 2, \ldots$ appear to be negligible. As shown in Table 1 , when $\alpha$ is very small these extra sources may have significant contributions to the primary distribution.

TABLE 1. Temperature $\tau$ at $\xi_{1}=0, \xi_{3}=0$, when

\begin{tabular}{rll}
$\alpha=0.05, \varepsilon=0.1$ & \\
$\ldots \ldots \ldots \ldots \ldots \ldots \ldots \ldots \ldots \ldots \ldots \ldots \ldots \ldots \ldots \ldots \ldots$ \\
$n$ & $\xi_{2}=0.1$ & $\xi_{2}=1.0$ \\
\hline 0 & 2.197 & 0.473 \\
-1 & 0.103 & 0.103 \\
1 & 0.0002 & 0.00017 \\
-2 & 0.074 & 0.074
\end{tabular}




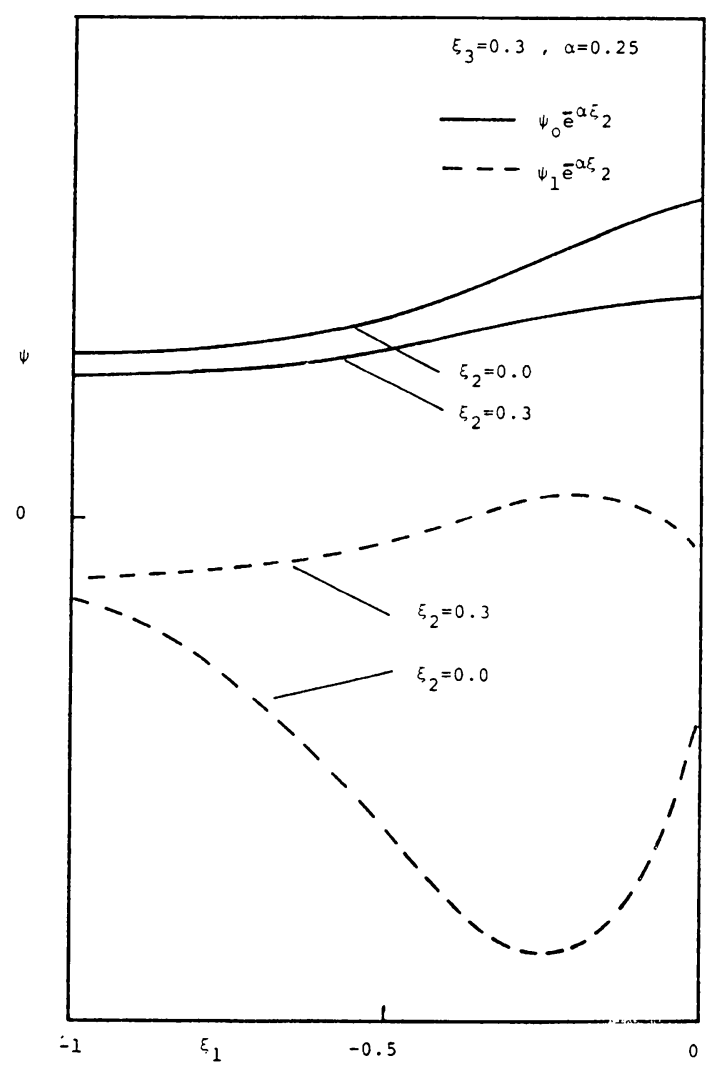

FIG. 3. Radial distribution of temperature.

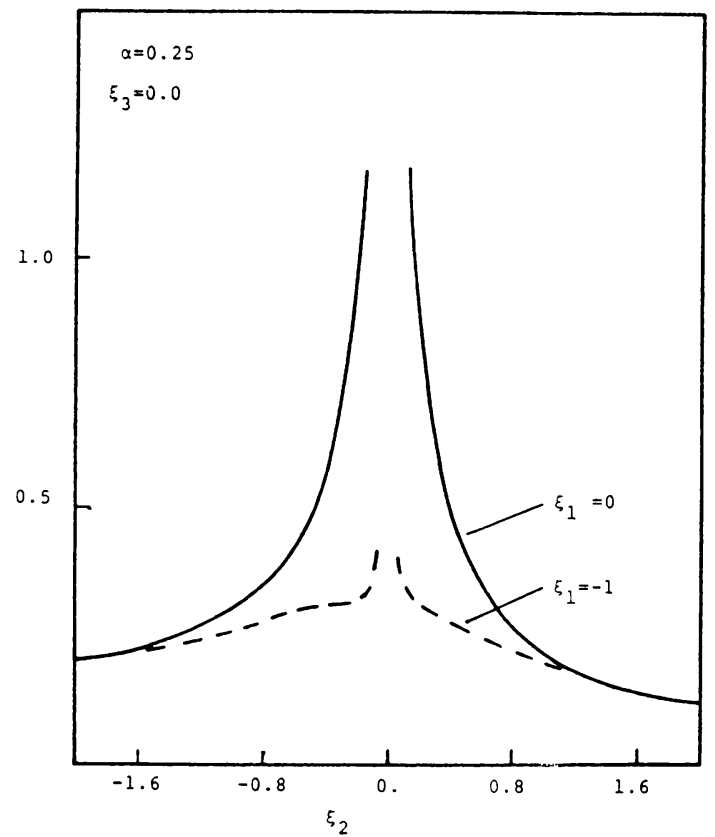

Fig. 4. Cumulative temperature on the surfaces of the cylinder due to a system of moving sources. 


\section{REFERENCES}

[1] A. A. Wells, The mechanics of brittle fracture, Welding Research 7, 34r-56r (1953)

[2] A. A. Wells, Heat flow in welding, Welding journal research supplement 31, 263s-267s (1952)

[3] H. A. Carslaw and J. C. Jaeger, Conduction of heat in solids, Second edition, Oxford Clarendon Press, 1959

[4] S. Vaidyanathan, A. F. Todaro and I. Finnie, Residual stresses due to circumferential welds, J.f Eng. Mat. and Tech. 95, 233-237 (Oct. 1973)

[5] S. Vaidyanathan, H. Weiss and I. Finnie, A further study of residual stresses in circumferential welds, Trans. ASME J. of Eng. Mat. and Tech. 95, 238-242 (Oct. 1973)

TWO FASCINATING, OUTSTANDING FILMSCOMPUTER GRAPHICS VISUALIZE DETERMINISTIC CHAOS THEORY

THE PLANAR DOUBLE PENDULUM

Uses the example of the planar double pendulum to demonstrate the principles of chaotic behavior. In the first section, computer graphics show periodic, quasiperiodic, and chaotic motion. In the second and third demonstrations, the computer generates a Poincaré section illustrating how periodic, quasiperiodic and chaotic motion can be distinguished, then shows these sections as they evolve under increasing energy levels.

28 minutes color

$16 \mathrm{~mm}$ sale $\$ 495$ or rental $\$ 35.00$ video sale $\$ 495$

\section{FLY LORENZ}

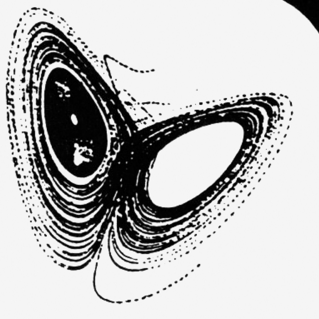

We fly with a red tetrahedron as test object along the trajectories of the Lorenz Attractor. The Boston skyline behind the Attractor represents the event horizon facilitating spatial orientation. Five experiments examine the qualities of the Lorenz Attractor, and the final scene takes us again on a camera trip over the Lorenz Attractor.

14 minutes color

$16 \mathrm{~mm}$ sale $\$ 325$ or rental $\$ 25.00$

video sale 4325

Request information about other algebra, geometry and topology films.

Available for purchase, preview or rental from:

INTERNATIONAL FILM BUREAU INC.

332 South Michigan Ave., Chicago, IL 60604 • (312) 427-4545 